\title{
Precision and Reproducibility of Quantitative Coronary Angiography with Applications to Controlled Clinical Trials
}

\section{A Sampling Study}

\author{
Robert H. Selzer, ${ }^{*}$ Cheryl Hagerty, ${ }^{\ddagger}$ Stanley P. Azen, ${ }^{\ddagger}$ Maria Siebes, ${ }^{\star}$ Paul Lee, ${ }^{\star}$ Anne Shircore, ${ }^{\star}$ David H. Blankenhorn, ${ }^{\ddagger}$ \\ and the Cholesterol Lowering Atherosclerosis Study Investigators and Staff \\ ${ }^{*}$ California Institute of Technology, Jet Propulsion Laboratory, Pasadena, California 91109, and ${ }^{\ddagger}$ Departments of Medicine and \\ Preventive Medicine, University of Southern California School of Medicine, Los Angeles, California 90033
}

\begin{abstract}
Most computer methods that quantify coronary artery disease from angiograms are designed to analyze frames recorded during the end-diastolic portion of the cardiac cycle. The purpose of this study was to determine if end diastole is the best portion of the cardiac cycle to sample, or if other sampling schemes produce more precise and/or reproducible estimates of coronary disease. 20 cinecoronary angiograms were selected at random from a controlled clinical trial testing the effects of plasma lipid lowering on atherosclerosis. Sampling schemes included sequential and random sampling of two to five frames within the complete cardiac cycle, systole, and diastole. Three vessel measures and percent stenosis were evaluated for each sampling scheme. From the sampling experiment, it was determined that sampling sequentially end diastole yielded the most precise estimates (i.e., exhibiting minimum variability within a cycle) of the vessel measures. With regard to reproducibility (i.e., similar values across cycles), sampling randomly within the cycle was best. Overall, the average diameter of a vessel segment was the most precise and the most reproducible of the measures. Sample size calculations are given for each of these measures under the best sampling scheme.
\end{abstract}

\section{Introduction}

Contrast angiography is the preferred method to provide insight about the progression/regression of coronary atherosclerosis in vivo (1). However, methodological difficulties exist in the objective evaluation of coronary angiograms. Quantitative computer-assisted methods have been developed with the goal of improving the precision of lesion measurement as an endpoint in clinical trials. Two basic types of quantitative analysis are presently used (2). In the first, coronary cine-angiograms are projected and vessel outlines are hand traced with a digitizing stylus. From these, lesion severity is calculated by computer $(3,4)$. In the second, selected frames from the cinefilm are digitized with a video camera and image processing techniques are used to locate the vessel edges and estimate the extent of lesions (5-9). Manual edge finding is subject to con-

Address reprint requests to Dr. David $\mathrm{H}$. Blankenhorn, Atherosclerosis Research Institute, University of Southern California School of Medicine, 2025 Zonal Avenue, Los Angeles, CA 90033.

Received for publication 1 June 1988 and in revised form 7 September 1988.

J. Clin. Invest.

(C) The American Society for Clinical Investigation, Inc.

$0021-9738 / 89 / 02 / 0520 / 07 \$ 2.00$

Volume 83, February 1989, 520-526 siderable interobserver and intraobserver variability due to the subjective process of locating the vessel edge within the penumbra appearing on the film $(3,10,11)$. Published estimates indicate that the precision of the automatic edge tracking method is about twice those of the manual tracing analysis $(6$, $9,12,13)$.

The performance of both methods suffers from inherent limitations to $\mathrm{x}$ ray imaging that reduce the contrast and sharpness of the image and complicate the comparison of repeated angiograms. These include effects in the imaging chain such as noise due to the inherent random nature of the $x$ ray attenuation and scattering process, and reduction in contrast from beam hardening and veiling glare in the image intensifier. Image digitization introduces noise in the video imaging chain and quantitization errors in the analog to digital conversion process. When angiography is applied to clinical trials that last several years, deterioration of the $x$ ray source with continued use, as well as change in the control of film development, can also contribute error.

Other major limiting factors are of biological origin, such as differences in vasomotor tone, vessel motion, myocardial blush, and filling of the vasa vasorum, and the uniformity of blood-contrast mixing. The latter has been shown to strongly affect both visual and automatic edge tracking $(9,12,14-16)$. Changes in size of the vessel image during the cardiac cycle have been attributed to differential magnification from vessel translation or rotation, pressure increase due to the injection itself, and arterial pressure pulsation. Periodic variations in vessel diameter of $5-10 \%$ from pulsation have been shown in both animals and man $(9,17-20)$. These are of the same order of magnitude as annual progression/regression rates of atherosclerosis $(21,22)$.

Image mottling in cine-angiograms, which is caused by a combination of $\mathrm{x}$ ray noise and nonuniform blood-contrast mixing, has been identified as a major source of error in quantitative analysis of a single angiographic frame $(8,11)$. Efforts to reduce these effects include spatial filtering (8), averaging of densitometric profiles from adjacent scanlines along a segment, or averaging of diameter profiles from sequential cineframes $(11,18,23)$. Periodic diameter variations due to arterial pressure pulsation, however, have received less attention. In most studies, a single end-diastolic frame is selected because vessel motion and time dependency of the arterial diameter is believed to be minimal during this phase of the cardiac cycle (3, 24-27). However, contrast concentration can be reduced by increased blood flow during diastole and this can reduce the precision of lesion measurement (11).

The potential for improving precision through measurement of multiple frames from an injection sequence has been pointed out by Spears (11) and Selzer (18). In theory, measure- 
ment error due to such random effects as quantum mottle and contrast mixing should be reduced by averaging measurements from multiple sequential frames. However, because the vessel changes during the cardiac cycle, results derived from frame averaging will depend on both the selected phase of the cardiac cycle and the number of frames used for averaging.

The purpose of this study was to determine the best sampling scheme to select frames from the cardiac cycle to quantify coronary disease. In a simulation study, different sampling schemes, using the complete cycle, systole, or diastole, were evaluated to determine which scheme(s) provide the most precise and reproducible estimates of vessel diameters and vessel stenosis. We defined a precise estimate as one that exhibits minimum variability within a cycle and a reproducible estimate as one that yields similar values across cycles. Precise determinations of the extent of disease within a single cardiac cycle are advantageous for one-time characterizations, as for example, in a case series when risk factors are correlated with a single angiogram. Reproducible determinations of disease characteristics across cardiac cycles are required for assessing treatment effects in controlled clinical trials. An optimal estimate would be one that is both precise and reproducible.

\section{Methods}

\section{Angiogram selection}

The angiograms studied in this experiment were obtained during the conduct of the Cholesterol Lowering Atherosclerosis Study (CLAS), ${ }^{1}$ a controlled clinical trial testing the effects of plasma lipid lowering on progression and regression of atherosclerosis (28). The trial subjects were 188 men, ages $40-59 \mathrm{yr}$ at the start of the study, who had undergone coronary bypass surgery before entry in the study. Examinations were performed by a single angiographer using the percutaneous femoral technique (29). All angiograms were filmed at 60 frames/s.

Selection of segments from the study film library was done by a cardiologist, the image processing computer operator, and a moderator who had participated in prior evaluation of the films by human readers. Segments were defined as being between major branches. This avoided problems with vessel edge tracking at branch junctions and also minimized the error associated with vessel taper. The criteria for selection of segments were: $(a)$ the target artery was adequately filled with contrast medium for two complete cardiac cycles ( $\sim 120$ frames), and $(b)$ the target segment was not obscured by crossing branches, overlying sternal sutures, or filling of a bypass graft.

Beginning with the earliest angiograms, a set of 34 films were selected using a sampling plan that randomized the eligibility of segments for consideration between subjects, examination years, and vessels. From these, 20 suitable coronary segments were identified which had unobstructed views of the left anterior descending artery (LAD; seven segments), right coronary artery (RCA; seven segments), and proximal circumflex (LCX; six segments). All but six segments were bypassed, and all but three had distal lesions, including complete obstruction. No specific effort was made to include normal segments since in this group of subjects, normal arteries would be difficult to find and verify.

1. Abbreviations used in this paper: ANOVA, analysis of variance; BS, $n$ sequential frames in end diastole; $\mathrm{CCV}$, cycle-to-cycle crefficient of variation; CLAS, Cholesterol Lowering Atherosclerosis Study; CR, $n$ random frames within the cycle; DAVG, average diameter; $D(90)$, 90th-percentile diameter; $\mathrm{D}(3)$, third-percentile diameter; $\mathrm{ED}, n$ sequential frames in beginning systole; FCV, frame-to-frame coefficient of variation; LAD, left anterior descending artery; LCX, proximal circumflex; RCA, right coronary artery; STEN, percent stenosis.
As shown in Table I, stenosis ranged from 13 to $62 \%$. Vessels with computer stenosis measurement of $<25-30 \%$ stenosis appeared normal to the eye.

The most common reason for rejecting a segment for this study was complete occlusion of the vessel (six segments) and the presence of overlapping vessels (four segments). For clinical applications, only three to five frames (rather than 120) would be needed for evaluation; hence, the inclusion rate would be much larger than 20 out of 34 .

\section{Equipment}

All angiograms were analyzed at the Biomedical Image Analysis Facility at the California Institute of Technology, Jet Propulsion Laboratory. The image processing system was a computer (PDP 11/45, Digital Equipment Corp., Marlboro, MA), an image array processor (IP 5500C; De Anza, Santa Barbara, CA), and a cine projector (M35C; Vanguard, Irvine, CA) coupled to a 525 line vidicon camera. A magnification of 2.5 was used, which corresponds to a pixel size of $15.6 \mu \mathrm{m}$ in the image plane.

\section{Frame selection and digitizing}

Only one vessel segment per patient was selected for image processing. The onset of systole and diastole in each cardiac cycle was identified by observation of motion in the vessel or a nearby branch, and the frames were indexed by the onset of the first systole. Each frame was digitized 32 times and averaged to reduce the effect of camera noise. All frames in two consecutive cycles for each patient were digitized, registered with respect to a common landmark such as a nearby vessel bifurcation, and stored on magnetic tape.

\section{Vessel edge tracking}

Segments to be analyzed were identified by the operator through indication of a number of points along the approximate vessel midline. Vessel edges were selected as the points of maximum gray-level gradient determined along a series of scanlines perpendicular to the midline. Gradient values for each scanline were computed as the derivative of a second degree polynomial fit to a moving window of gray scale values (30).

The number of points in the moving window was adjusted from 5 to 13 according to the average width of the previous five vessel diameters to avoid overestimation of narrow sections. The computed intensity gradients were smoothed over three to seven points, depending on the size of the moving window. To reduce the effect of multiple gradient maxima/minima in a single scanline due to branches or nearby vessels, an exponential weighting function centered at a distance from the midline corresponding to the prior edge location was applied to the smoothed gradient values. The coordinates of the maximum weighted gradient was selected as an edge point.

After a selected segment had been tracked in this manner, a new midline was calculated and the edge search was repeated to reduce effects of operator-associated variability in selecting the initial midline. All diameters were converted to millimeters by a scaling factor obtained from known dimensions of the catheter. After complete analysis of the first frame for a given patient, the next frame was read from tape and a current midline passed on to the next frame for subsequent automatic analysis. After all frames for a patient had been processed, an optional editing procedure allowed the operator to correct erroneous edges. The number of frames analyzed per cycle for each study subject varied according to heart rate because the film speed was constant (Table I).

\section{Measures}

Three vessel edge measures $D(3), D(90)$, and average diameter (DAVG) were computed for each frame from the diameter profile along the analyzed segment. $D(3)$, the third-percentile diameter $(97 \%$ of diameters in the profile are larger), was used as an estimate of the narrowest point in the segment. $D(90)$, the 90th-percentile diameter (only $10 \%$ of the diameters in the segment are larger), was used as an estimate of the normal reference diameter of the segment. DAVG was 
Table I. Description of Study Sample

\begin{tabular}{|c|c|c|c|c|c|c|c|c|c|}
\hline \multirow[b]{3}{*}{$\begin{array}{l}\text { Vessel } \\
\text { type }\end{array}$} & \multicolumn{4}{|c|}{ No. of frames } & \multirow[b]{3}{*}{$\begin{array}{l}\text { Segment } \\
\text { length }\end{array}$} & \multirow{2}{*}{\multicolumn{4}{|c|}{ Means* }} \\
\hline & \multicolumn{2}{|c|}{ Cycle 1} & \multicolumn{2}{|c|}{ Cycle 2} & & & \multirow[b]{2}{*}{$\mathrm{D}(90)$} & & \multirow[b]{2}{*}{ STEN } \\
\hline & Systole & Diastole & Systole & Diastole & & D (3) & & DAVG & \\
\hline & & & & & \multicolumn{4}{|c|}{$m m$} & $\%$ \\
\hline LAD & 23 & 27 & 24 & 27 & 9.1 & 1.9 & 2.8 & 2.4 & 30.9 \\
\hline LAD & 15 & 17 & 15 & 16 & 10.9 & 1.6 & 2.1 & 1.9 & 26.0 \\
\hline LAD & 20 & 41 & 20 & 40 & 18.4 & 2.0 & 3.6 & 2.9 & 45.1 \\
\hline LAD & 21 & 33 & 22 & 30 & 22.8 & 1.8 & 4.0 & 2.9 & 54.5 \\
\hline LAD & 26 & 27 & 28 & 25 & 11.9 & 1.7 & 2.4 & 2.1 & 31.3 \\
\hline LAD & 21 & 25 & 20 & 26 & 21.6 & 1.8 & 2.8 & 2.4 & 34.7 \\
\hline LAD & 15 & 32 & 15 & 32 & 10.3 & 1.4 & 3.3 & 2.6 & 58.0 \\
\hline $\mathrm{RCA}$ & 26 & 31 & 26 & 31 & 20.5 & 2.7 & 3.1 & 2.9 & 13.4 \\
\hline $\mathrm{RCA}$ & 19 & 36 & 19 & 34 & 19.2 & 1.5 & 4.0 & 2.7 & 62.1 \\
\hline $\mathrm{RCA}$ & 16 & 22 & 16 & 23 & 13.8 & 1.2 & 2.8 & 2.2 & 56.6 \\
\hline RCA & 23 & 36 & 24 & 37 & 29.4 & 3.4 & 5.1 & 4.5 & 32.9 \\
\hline $\mathrm{RCA}$ & 18 & 33 & 17 & 30 & 16.5 & 2.5 & 5.0 & 3.8 & 50.5 \\
\hline $\mathrm{RCA}$ & 20 & 29 & 21 & 27 & 8.5 & 2.0 & 3.2 & 2.5 & 35.6 \\
\hline $\mathrm{RCA}$ & 28 & 35 & 28 & 35 & 11.7 & 1.0 & 2.1 & 1.6 & 50.5 \\
\hline LCX & 15 & 35 & 17 & 34 & 17.5 & 2.5 & 3.9 & 3.4 & 35.4 \\
\hline LCX & 15 & 17 & 15 & 17 & 16.4 & 3.8 & 4.7 & 4.3 & 18.0 \\
\hline LCX & 19 & 29 & 20 & 28 & 19.9 & 1.4 & 2.6 & 2.2 & 46.1 \\
\hline LCX & 21 & 21 & 21 & 22 & 16.6 & 1.3 & 2.4 & 2.0 & 44.6 \\
\hline LCX & 15 & 23 & 15 & 23 & 14.4 & 1.7 & 3.0 & 2.3 & 41.1 \\
\hline LCX & 18 & 32 & 17 & 27 & 13.4 & 2.3 & 3.3 & 2.8 & 31.1 \\
\hline
\end{tabular}

* Means calculated for scheme ED, $n=3$ (see text).

defined as the sum of all diameters divided by the total number of scanlines per segment. Percent stenosis was defined as:

$$
\text { STEN }=100[1-D(3) / D(90)] .
$$

Plots of the four measures from a segment of the circumflex artery of two subjects are shown in Figs. 1 and 2. From top to bottom, the plots show D(90), DAVG, D(3), and STEN, respectively. (The darker line in each plot presents results of a five-point smoothing filter.) The circumflex artery in Fig. 1 was not bypassed while that in Fig. 2 had received a bypass graft. The cyclic change in diameter shown in the unbypassed vessel is frequently not apparent in bypassed vessels.

\section{Sampling schemes}

We report on three sampling schemes for selecting $n$ frames within different phases of a single cycle: $\mathrm{ED}, n$ sequential frames in end diastole; BS, $n$ sequential frames in beginning systole; and CR, $n$ random frames within the cycle.

For ED, the last frame in diastole is selected as the starting frame of the sample, and the next $n-1$ frames are sampled sequentially moving back through diastole. For BS, the first frame of systole is selected as the starting frame of the sample, and the next $n-1$ frames are sampled sequentially moving forward through systole. For CR, all $n$ frames are chosen at random with no frame being chosen more than once.

Nine sampling schemes in other parts of the cardiac cycle, such as middiastole and anywhere in systole, were tested. However, ED, BS, and CR were selected for detailed examination because the results for these schemes bracket those for all other sampling schemes.

Processing of $n=2,3,4$, and 5 frames was evaluated. Processing more than five frames was considered to be too time consuming for current practice (the average time to process five frames, once the initial frame was selected, was $4 \mathrm{~min}$ ). An additional reason for not processing more than five frames was the concern that periodic dimensional changes in the vessel image during the cardiac cycle due to pulsation, differential magnification of the vessel, and perspective changes relative to the $x$ ray imaging system would increase the variability of dimensional measurements for large $n$.
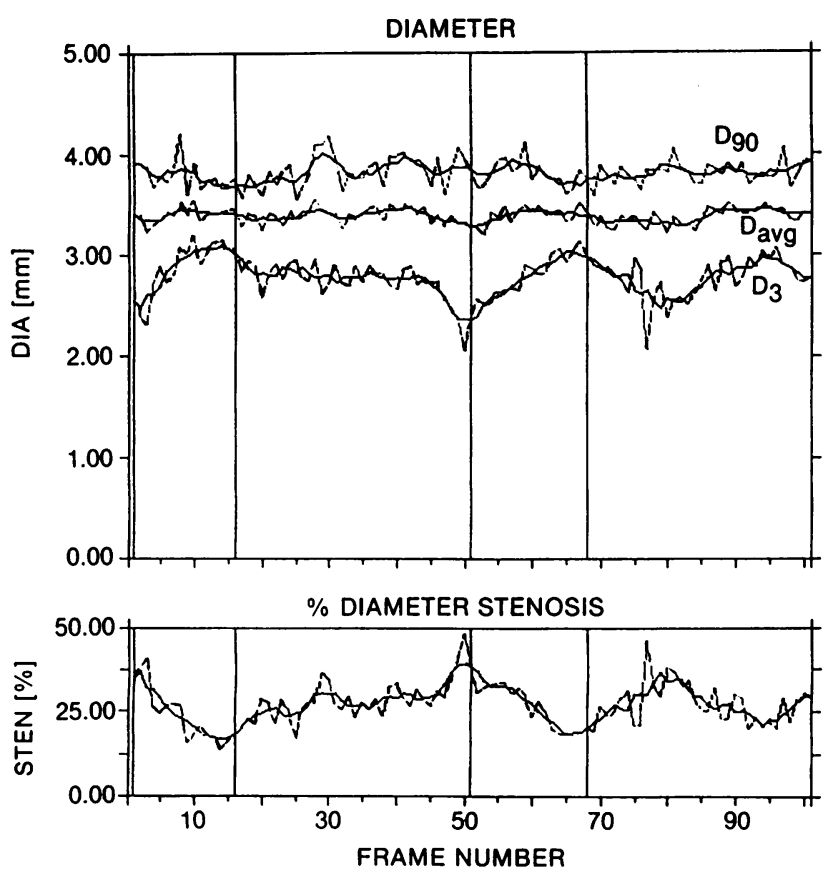

Figure 1. Plot of diameter and stenosis measurements over two cardiac cycles for a segment of an unbypassed circumflex artery. From top to bottom, plots show D(90), DAVG, D(3), and STEN. Darker lines show diameters after five-point averaging filter. 

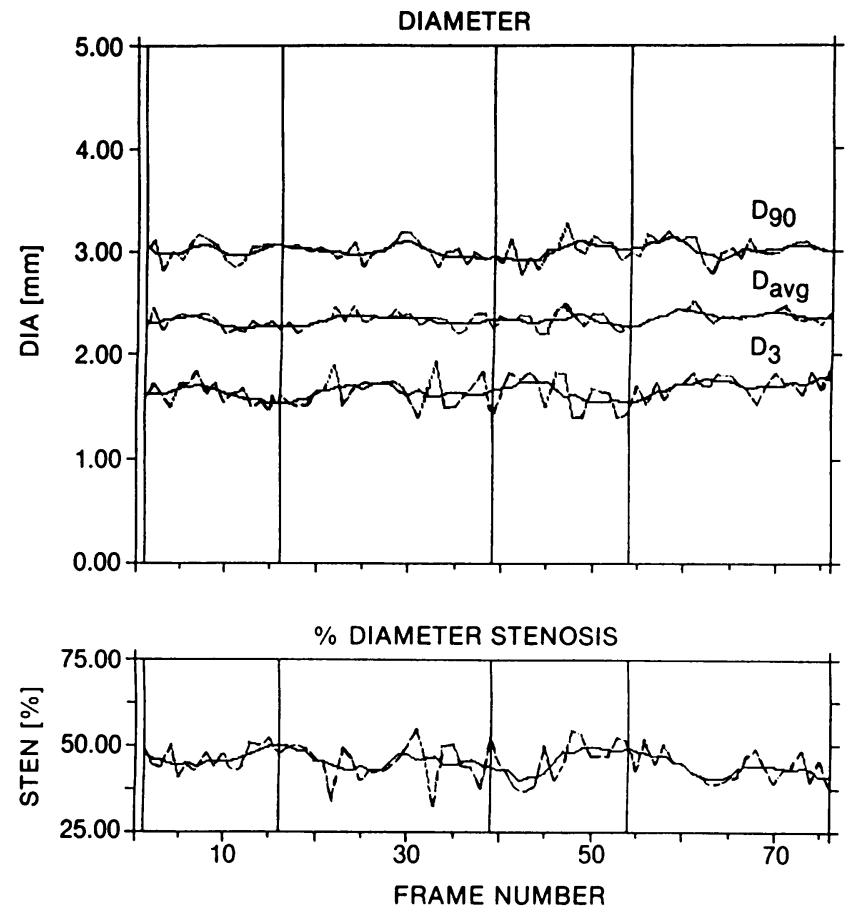

Figure 2. Similar diameter plots as shown in Fig. 1 except measured segment is a bypassed circumflex artery.

\section{Statistics of precision and reproducibility}

Frame-to-frame coefficient of variation (FCV). The precision of an estimate of a vessel measure was defined as the FCV. This quantity was calculated for each subject, measure ( $D(3), D(90)$, DAVG, and STEN), sample size ( $n=2,3,4$, and 5$)$, and sampling scheme (ED, BS, and $\mathrm{CR}$ ).

To calculate FCV for a given subject (subject 1, for example), we selected a measure (e.g., DAVG), sample size (e.g., $n=3$ ), and sampling scheme (e.g., ED). The three end-diastolic frames in this situation are $2.497,2.386$, and 2.419 for cycle 1 , and $2.496,2.455$ and 2.445 for cycle 2 . We then calculated for each cycle the frame-to-frame means, $\bar{x}_{1}$ and $\bar{x}_{2}$; the standard deviations, $\mathrm{SD}_{1}$ and $\mathrm{SD}_{2}$, and coefficients of variation, $\mathrm{CV}_{1}=100\left(\mathrm{SD}_{1} / \overline{\mathrm{x}}_{1}\right)$ and $\mathrm{CV}_{2}=100\left(\mathrm{SD}_{2} / \overline{\mathrm{x}}_{2}\right)$. For this subject, $\overline{\mathrm{x}}_{1}=2.434, \overline{\mathrm{x}}_{2}=2.465, \mathrm{SD}_{1}=0.057, \mathrm{SD}_{2}=0.027, \mathrm{CV}_{1}=2.34$, and $\mathrm{CV}_{2}=1.10$. Then, FCV was calculated as the average coefficient of variation for the two cycles, namely, $F C V=\left(C_{1}+C V_{2}\right) / 2$. For the above example, $\mathrm{FCV}=1.72$.

For scheme CR, the value of FCV depended on the random selection of all frames. To minimize the effect of the random selection process, the process of selecting frames and computing FCV was repeated 50 times, and the average of the 50 trials was used to represent FCV for each subject.

Cycle-to-cycle coefficient of variation (CCV). Reproducibility was defined as the CCV. This quantity was calculated for each subject, measure (D(3), D(90), DAVG, and STEN), sample size ( $n=2,3,4$, and 5), and sampling scheme (ED, BS and CR). For each subject, measure, sample size and sampling scheme, the means $\bar{x}_{1}$ and $\bar{x}_{2}$ were computed for each of the two cardiac cycles. The average $\bar{x}$ and the SD were calculated as: $\overline{\mathbf{x}}=\left(\overline{\mathbf{x}}_{1}+\overline{\mathbf{x}}_{2}\right) / 2$, and $\mathrm{SD}=\sqrt{\left[\left(\overline{\mathbf{x}}_{1}-\overline{\mathbf{x}}\right)^{2}+\left(\overline{\mathbf{x}}_{2}-\overline{\mathbf{x}}\right)^{2}\right]}$. The coefficient of variation, CCV, of the two means was then computed as: $\mathrm{CCV}=100 \mathrm{SD} / \overline{\mathrm{x}}$.

For the above example, $\overline{\mathrm{x}}=2.450, \mathrm{SD}=0.042$, and $\mathrm{CCV}=1.71$.

To reduce variability due to the random selection of frames for method CR, the sampling experiment was also replicated 50 times. The resulting data to be analyzed consisted of the average of 50 values of CCV.

\section{Statistical analysis}

To identify sampling schemes that produced precise estimates of each vessel measure, the frame-to-frame statistic, FCV, was used. To identify the sampling scheme that produced reproducible estimates of each vessel measure, the between-cycle statistic, CCV, was used. For each sample size, FCV and CCV were analyzed as a $20 \times 3$ randomized blocks analysis of variance (ANOVA) (blocking factor = subject, treatment factor $=$ sampling scheme). Because of the nonnormality of the data, these data were analyzed using the nonparametric randomized blocks ANOVA method of Friedman-Kendall-Smith (31). Pairwise comparisons between sampling schemes were made using a multiple-comparison procedure of Friedman-Kendall-Smith (31).

Data were also analyzed by vessel type (LAD, LCX, RCA). Finally, because cinecoronary angiograms are also exposed at 30 frames/s instead of $60 / \mathrm{s}$ as selected for the CLAS study, analyses were also carried out using information for every other frame to mimic this situation.

\section{Results}

Because analyses by vessel type and by frame speed produced similar results, we present results for all three vessel types combined and for a speed of 60 frames/s (32). Table II presents summary statistics for the measures $D(3), D(90)$, DAVG and STEN. Shown in the table are the two coefficients of variation (FCV and CCV) for each of the sampling schemes and four values of $n$. For each $n$, the minimum values of FCV and CCV are starred $(*)$.

FCV was examined to determine which scheme produced the most precise estimate of each measure within a single cycle. For each measure, scheme ED, sequential end-diastole, had the minimum FCV for all $n$. Scheme ED was significantly different from scheme $C R$, random within cycle $(P<0.001)$.

$\mathrm{CCV}$ was examined to determine which sampling scheme produced the most reproducible estimates across cycles. No single sampling scheme uniformly produced a minimum CCV for all measures and all $n$. For DAVG, scheme ED produced the minimum $\mathrm{CCV}$ when $n<5$.

Because there was a decreasing trend in $\mathrm{CCV}$ with increasing $n$, the percent change in CCV due to increasing the sample size from 2 to 3,3 to 4 , and 4 to 5 , was examined for each measure. For $\mathrm{D}(3), \mathrm{D}(90)$ and STEN, no optimal $n$ could be determined. For DAVG the percent change in CCV was decreased when sampling three frames instead of two, but did not improve when sampling four or five frames.

\section{Discussion}

From the results of this sampling experiment, it appears that for estimating all four measures, sampling sequentially enddiastole (scheme ED) yielded the most precise estimates of the vessel measures within a single cardiac cycle. The fully random sampling scheme (scheme CR) produced significantly less precise estimates $(P<0.001)$. Other sequential schemes that we considered, but are not detailed here, include: $(a)$ randomly selecting a starting frame within diastole, and then sampling sequentially in the direction containing the most diastolic frames; $(b)$ randomly selecting a starting frame at mid-diastole \pm one-fourth the diastolic interval, and then sampling sequentially in the direction containing the most diastolic frames; $(c)$ randomly selecting a starting frame within systole, 
Table II. Comparison of Sampling Schemes for Different Sample Sizes for the Measures D(3), D(90), DAVG, and STEN

\begin{tabular}{|c|c|c|c|c|c|c|}
\hline \multirow[b]{2}{*}{ Measure } & \multirow[b]{2}{*}{ Statistic } & \multirow[b]{2}{*}{ Scheme } & \multicolumn{4}{|c|}{ No. of frames } \\
\hline & & & 2 & 3 & 4 & 5 \\
\hline \multirow[t]{6}{*}{$D(3)$} & FCV & ED & $3.5^{*}$ & $4.3^{*}$ & $5.0^{*}$ & $5.4^{*}$ \\
\hline & & BS & 5.6 & 6.2 & 6.1 & 6.5 \\
\hline & & $\mathrm{CR}$ & 6.1 & 6.5 & 6.8 & 6.9 \\
\hline & $\mathrm{CCV}$ & ED & 4.9 & 4.8 & 4.2 & 3.9 \\
\hline & & BS & 5.2 & $3.3^{*}$ & 3.1 & 3.1 \\
\hline & & CR & $4.1^{*}$ & 3.4 & $3.0^{*}$ & $2.7^{*}$ \\
\hline \multirow[t]{6}{*}{$\mathrm{D}(90)$} & FCV & ED & $1.8^{*}$ & $2.3^{*}$ & $2.5^{*}$ & $2.7^{*}$ \\
\hline & & BS & 2.5 & 3.0 & 3.3 & 3.4 \\
\hline & & $\mathrm{CR}$ & 3.0 & 3.2 & 3.5 & 3.5 \\
\hline & $\mathrm{CCV}$ & ED & 2.0 & 1.9 & 1.8 & 1.6 \\
\hline & & BS & $1.6^{*}$ & 2.0 & 1.8 & 1.7 \\
\hline & & $\mathrm{CR}$ & 2.0 & $1.7^{*}$ & $1.5^{*}$ & $1.4^{*}$ \\
\hline \multirow[t]{6}{*}{ DAVG } & FCV & ED & $1.5^{*}$ & $1.9^{*}$ & $2.1^{*}$ & $2.2^{*}$ \\
\hline & & BS & 2.1 & 2.2 & 2.4 & 2.5 \\
\hline & & CR & 2.5 & 2.7 & 2.9 & 2.9 \\
\hline & $\mathrm{CCV}$ & ED & $1.6^{*}$ & $1.3^{*}$ & $1.3^{*}$ & 1.4 \\
\hline & & BS & 2.5 & 2.2 & 1.6 & 1.4 \\
\hline & & $\mathrm{CR}$ & 1.7 & 1.5 & 1.4 & $1.3^{*}$ \\
\hline \multirow[t]{6}{*}{ STEN } & FCV & ED & $7.6^{*}$ & $9.6^{*}$ & $10.2^{*}$ & $10.6^{*}$ \\
\hline & & BS & 10.4 & 11.5 & 11.1 & 12.1 \\
\hline & & CR & 10.8 & 11.5 & 12.0 & 12.3 \\
\hline & $\mathrm{CCV}$ & ED & 8.8 & 8.2 & 7.6 & 6.8 \\
\hline & & BS & 7.4 & 6.1 & 5.3 & 5.6 \\
\hline & & $\mathrm{CR}$ & $7.2^{*}$ & $5.7^{*}$ & $5.0^{*}$ & $4.4^{*}$ \\
\hline
\end{tabular}

BS, Sequential in beginning systole; ED, Sequential end diastole; and CR, Random within cycle.

* Minimum value of FCV or CCV among ED, BS, and CR.

and then sampling sequentially in the direction containing the most systole frames; and $(d)$ randomly selecting a starting frame anywhere within the cycle, and then sampling sequentially. Sampling within middiastole was competitive with scheme ED; sampling within systole or at random within the cycle was generally worse (32). Thus, for correlation of any or all of the four measures in a single angiogram with risk factor data for description of a case series, sequential frames averaged in end diastole appears optimal; sampling sequentially within middiastole is almost as good (32).

With regard to reproducibility, the random sampling scheme (scheme CR) was slightly better than scheme ED for all measures except for DAVG. Other random schemes that we considered, but are not detailing here, include: $(a)$ random sampling within diastole, $(b)$ random sampling within middiastole \pm one-fourth the diastolic interval; and $(c)$ randomly sampling within systole. These random sampling schemes were competitive but less reproducible (32). For measurement of DAVG, scheme ED (sequential, end diastole) produced estimates that are both precise within a cycle, and reproducible across cycles. Further, the optimal number of frames was determined to be three; sampling more than three frames did not decrease the CCV.

DAVG appears to be an attractive measurement for both clinical trials and for one time case description. The precision and reproducibility of DAVG are better than those of $D(3)$, $\mathrm{D}(90)$, or STEN because DAVG represents the average of all edge-to-edge diameters measured within a segment, whereas
$D(3)$ and $D(90)$ are determined by a small subset of these diameters. DAVG provides an integrated measure of arterial dimensions within a vessel segment.

Our results confirm and expand the conclusions of previous sampling studies. Ellis found DAVG to be the best overall measure (24). Sandor (17) found an average 5\% variation in diameter measurements made in 14 consecutive cardiac cycles in dogs. Frame-to-frame variation was believed mainly due to incomplete contrast mixing. Reiber et al. (27) studied the variability of coronary artery diameter measurements in end diastole in 38 angiograms by comparing the measured diameter of the best end-diastole frame with that of the three preceding and following frames. They found the SD of the difference of pairs of measurements of minimum diameter (similar to $D(3)$ ) to vary from 0.19 to $0.24 \mathrm{~mm}$, depending on which frame was compared with the center frame. The SD for reference diameter (similar to $\mathrm{D}(90)$ ) varied from 0.09 to $0.15 \mathrm{~mm}$. The separation between frames was not found to be significant. When the pairs of frames from the same phase of sequential cardiac cycles were compared, the SD was $0.21 \mathrm{~mm}$ for minimum diameter and $0.18 \mathrm{~mm}$ for an adjacent reference diameter. They concluded that frame selection during end diastole was not critical, and indicated that contrast mixing was the major source of measurement artifact. One reassuring conclusion of our study is that if a vessel cannot be analyzed during end diastole, for example, because of overlapping structures, then mid diastole or a different period of the cardiac cycle may be selected with only a small cost in precision. 
We have estimated the sample size requirements for a clinical trial designed to detect a differential treatment effect in DAVG of $2 \%$ using method ED with and without frame averaging. To calculate sample sizes, we needed an estimate of the correlation between pre- and posttreatment determinations of DAVG to compute the estimated variance in the change in DAVG. We estimated this correlation from the two successive cycles sampled in each subject realizing that these correlations would overestimate the correlation obtained after a typical treatment interval. The resulting correlations, variances and sample sizes are given in Table III for $n=1,3$, and 5. For DAVG, there is a threefold decrease in sample size requirements when averaging three frames, as compared with measuring just one; averaging five frames does not reduce the sample size requirements.

This large reduction in sample size requirements was attributable in part to the near perfect correlation between preand postdeterminations of DAVG. Under actual conditions of a clinical trial, this correlation would be smaller, and sample size requirements (or effect sizes) would be much larger. For example, with 100 subjects per group, and using three frames to estimate DAVG, one could detect a $17 \%$ therapy effect if the correlation were 0.9 , a $29 \%$ effect if the correlation were 0.7 , and a $37 \%$ effect if the correlation were 0.5 .

If $\mathrm{D}(3)$, the flow-limiting diameter in coronary segments, or STEN, a computer surrogate for the most common measure used by human readers, is selected as an endpoint, more subjects will need to be entered to detect equivalent therapy effects (Table III). However, larger therapy effects could be detected in $\mathrm{D}(3)$ and STEN using the sample sizes determined for DAVG. For example, when averaging three frames with a sample of 39 patients/group, one could detect a 7\% differential effect in $D(3)$, and a $12 \%$ differential effect in STEN. Note that STEN does not exactly reproduce human evaluations because it uses information obtained in only one plane, and is always the ratio of diameters in a single vessel segment. Human readers combine information from several planes and are

Table III. Comparison of Sample Size Requirement for Detecting a $2 \%$ Change in DAVG, D(3) and STEN between Two Groups Using Sampling Scheme ED (Significance Level $=0.05$, Power = 0.8)

\begin{tabular}{clccc}
\hline & & \multicolumn{3}{c}{ No. of frames $(n)$} \\
\cline { 3 - 5 } Measure & Scheme & 1 & 3 & 5 \\
\hline \multirow{2}{*}{ DAVG $(\mathrm{mm})$} & Corr.* & 0.990 & 0.997 & 0.997 \\
& SD & 0.146 & 0.086 & 0.084 \\
& $n /$ group & 114 & 39 & 38 \\
D(3) $(\mathrm{mm})$ & Corr. & 0.95 & 0.98 & 0.98 \\
& SD & 0.311 & 0.225 & 0.181 \\
& $n /$ group & 951 & 494 & 322 \\
& Effect & $6 \%$ & $7 \%$ & $6 \%$ \\
STEN $(\%)$ & Corr. & 0.85 & 0.92 & 0.95 \\
& SD & 10.4 & 7.6 & 6.1 \\
& $n /$ group & 2,670 & 1,421 & 913 \\
& Effect & $12 \%$ & $12 \%$ & $10 \%$ \\
& & & & \\
\hline
\end{tabular}

\footnotetext{
* Correlation between two successive cycles.
}

‡Effect calculated using $n$ /group specified for DAVG. more flexible in selecting the diameter to be combined into the ratio used to calculate percent stenosis.

This study of films drawn at random from a clinical trial that took over six years to complete sampled both short- and long-term influences on angiographic measurements. It extends what has been learned by previous researchers who analyzed error in films collected over only short periods and has shown that frame averaging significantly reduces measurement error. For angiographic trials planned to test a predetermined therapy effect, substitution of frame averaging for single frame measurement will allow a reduction in the number of subjects required. For angiographic trials in which the number of subjects is set by other considerations, substitution of frame averaging for single frame measurement will allow smaller therapy effects to be detected.

\section{Acknowledgments}

This study was supported in part by the National Heart, Lung, and Blood Institute Program Project grant HL-23619.

\section{References}

1. Malinow, M. R. Regression of atherosclerosis in humans: fact or myth? Editorial. 1981. Circulation. 64:1-3.

2. Reiber, J. H. C., C. J. Kooijman, C. J. Slager, J. J. Gerbrands, J. C. H. Schuurbiers, A. Denboer, W. Wijns, and W. Serruys. 1984. Computer-assisted analysis of the severity of obstructions from coronary cineangiography: a methodological review. Automedica (Lond.). 5:219-238.

3. Brown, B. G., E. Bolson, M. Frimer, and H. T. Dodge. 1977. Quantitative coronary arteriography. Estimation of dimensions, hemodynamic resistance, and atheroma mass of coronary artery lesions using the arteriogram and digital computation. Circulation. 55:329337.

4. Kirkeeide, R. L., B. Wuesten, and M. Gottwik. 1981. Computer assisted evaluation of angiographic findings. In Thrombose Und Atherogenese. K. Breddin, editor. Gerhard Witzstrock Verlag, BadenBaden/Cologne/New York. 414-417.

5. Booman, F., J. H. C. Reiber, J. J. Gerbrands, C. J. Slager, J. C. H. Schuurbiers, and G. T. Meester. 1979. Quantitative analysis of coronary occlusions from coronary cine-angiograms. In Computers in Cardiology. IEEE Computer Society, Long Beach, CA. 177-180.

6. Kirkeeide, R. L., P. Fung, R. W. Smalling, and K. L. Gould. 1982. Automated evaluation of vessel diameter from arteriograms. In Computers in Cardiology. IEEE Computer Society, Long Beach, CA. 215-218.

7. Ledbetter, D. C., R. H. Selzer, R. M. Gordon, D. H. Blankenhorn, and M. E. Sanmarco. 1978. Computer quantitation of coronary angiograms. Noninvasive Cardiovasc. Meas. 167:27-36.

8. Sanders, W. J., E. L. Alderman, and D. C. Harrison. 1979. Coronary artery quantification using digital imaging processing techniques. In Computers in Cardiology, IEEE Computer Society, Long Beach, CA. 15-19.

9. Spears, J. R., T. Sandor, A. V. Als, M. Malagold, J. E. Markis, W. Grossman, J. R. Serur, and S. Paulin. 1983. Computerized image analysis for quantitative measurement of vessel diameter from cineangiograms. Circulation. 68:453-461.

10. Gould, K. L. 1985. Quantification of coronary artery stenosis in vivo. Circ. Res. 57:341-353.

11. Spears, J. R. 1986. Quantitation of anatomic coronary stenosis severity: limitations of accuracy. In State of the Art in Quantitative Coronary Arteriography. J. H. C. Reiber and P. W. Serruys, editors. Martinus Nijhoff, Dordrecht, Netherlands. 103-124. 
12. Reiber, J. H. C., C. J. Kooijman, C. J. Slager, J. J. Gerbrands, J. C. H. Schuurbiers, A. den Boer, W. Wijns, P. W. Serruys, and P. G. Hugenholtz. 1984. Coronary artery dimensions from cineangiogramsmethodology and validation of a computer-assisted analysis procedure. IEEE (Inst. Electr. Electron. Eng.) Trans. Med. Imag. MI-3:131-141.

13. Spears, J. R., T. Sandor, A. Als, M. Malagold, J. Markis, and S. Paulin. 1981. Accuracy of computer vs. visual measurement of vessel diameter from cineangiograms. Circulation. 64(Suppl. IV):130.

14. Crawford, D. W., E. S. Beckenbach, D. H. Blankenhorn, and S. H. Brooks. 1974. Grading of coronary atherosclerosis: comparison of a modified IAP visual grading method and a new quantitative angiographic technique. 1974. Atherosclerosis. 19:231-241.

15. Jaques, P., F. DiBianca, S. Pizer, F. Kohout, L. Lifshitz, and D. Delany. 1985. Quantitative coronary fluorography: computer vs. human estimation of vascular stenoses. Invest. Radiol. 20:45-52.

16. Siebes, M., M. Gottwik, and M. Schlepper. 1982. Qualitative and quantitative experimental studies on the evaluation of model coronary arteries from angiograms. In Computers in Cardiology. IEEE Computer Society, New York. 211-214.

17. Sandor, T., J. R. Spears, and S. Paulin. 1981. Densitometric determination of changes in the dimensions of coronary arteries. SPIE Digital Radiography 314:263-272.

18. Selzer, R. H. A second look at quantitative coronary angiography: some unexpected problems. 1986. In State of the Art in Quantitative Coronary Arteriography. J. H. C. Reiber and W. P. Serruys, editors. Martinus Nijhoff, Dordrecht, Netherlands. 125-144.

19. Tomoike, H., H. Ootsubo, K. Sakai, Y. Kikuchi, and M. Nakamura. 1981. Continuous measurement of coronary artery diameter in situ. Am. J. Physiol. 240:H73-H79.

20. Vatner, S. F., A. Pasipoularides, and I. Mirsky. 1984. Measurement of arterial pressure-dimension relationships in conscious animals. Ann. Biomed. Eng. 12:521-534.

21. Arntzenius, A. C., D. Kromhout, J. Barth, J. H. C. Reiber, A. V. G. Bruschke, B. Buis, C. M. van Gent, N. Kempen-Voogd, S. Strikwerda, and E. A. van der Velde. 1985. Diet, lipoproteins, and the progression of coronary atherosclerosis. The Leiden Intervention Trial. N. Engl. J. Med. 312:805-811.

22. Cashin, W. L., S. H. Brooks, D. H. Blankenhorn, R. H. Selzer, M. E. Sanmarco, and B. Benjauthrit. 1984. Computerized edge track- ing and lesion measurement in coronary angiograms. Atherosclerosis. 52:295-300.

23. Spears, J. R., T. Sandor, A. V. Als, M. Malagold, J. E. Markis, W. Grossman, J. R. Serur, and W. Paulin. 1983. Computerized image analysis for quantitative measurement of vessel diameter from cineangiograms. Circulation. 68:453-461.

24. Ellis, S., W. Sanders, C. Goulet, R. Miller, K. C. Cain, J. Lesperance, M. G. Bourassa, and E. L. Alderman. 1986. Optimal detection of the progression of coronary artery disease: comparison of methods suitable for risk factor intervention trials. Circulation. 74:1235-1242.

25. Sanz, M. L., G. B. J. Mancini, M. T. LeFree, J. K. Mickelson, M. R. Starling, R. A. Vogel, and E. J. Topol. 1987. Variability of quantitative digital subtraction angiography before and after percutaneous transluminal coronary angioplasty. Am. J. Cardiol. 60:55-60.

26. Reiber, J. H. C., P. W. Serruys, C. J. Kooijman, W. Wijns, C. J. Slager, J. J. Gerbrands, J. C. Schuurbiers, A. den Boer, and P. G. Hugenholtz. 1985. Assessment of short, medium and long-term veriation in arterial dimensions from computer assisted quantitation of coronary angiograms. Circulation. 71:280-288.

27. Reiber, J. H. C., P. van Eldik, C. J. Kooijman, and P. W. Serruys. 1987. Time-dependency in coronary obstruction measurements. Eur. Heart J. 8(Suppl. 2): 182.

28. Blankenhorn, D. H., S. A. Nessim, R. L. Johnson, M. E. Sanmarco, S. P. Azen, and L. Cashin-Hemphill. 1987. Beneficial effects of combined colestipol-niacin therapy on coronary atherosclerosis and coronary venous bypass grafts. JAMA (J. Am. Med. Assoc.) 257:32333240 .

29. Blankenhorn, D. H., R. L. Johnson, S. A. Nessim, S. P. Azen, M. E. Sanmarco, R. H. Selzer, and the CLAS Investigators and Staff. 1988. Cholesterol Lowering Atherosclerosis Study (CLAS): design, methods, and baseline results. Controlled Clin. Trials. 8:354-387.

30. Selzer, R. H., D. H. Blankenhorn, S. H. Brooks, D. W. Crawford, and L. Cashin. 1982. Computer assessment of atherosclerosis from angiographic images. IEEE (Inst. Electr. Electron. Eng.) Trans. Nucl. Sci. 29:1198-1207.

31. Hollander, M., and D. Wolfe. 1973. Nonparametric Statistical Methods. John Wiley \& Sons, New York. 182-193.

32. Hagerty, C. 1988. A sampling study in quantitative coronary angiography with applications to controlled clinical trials. M. S. Thesis, Division of Biometry, University of Southern California. 